\title{
Análise da presença de grupos estratégicos diversificados em empresas brasileiras de capital aberto
}

\author{
Analysis of the presence of diversified strategy groups in Brazilian traded companies
}

Hanna Tatarchenko Welgacz ${ }^{[a]}$, Isabel Cristina da Costa Domingues ${ }^{[b]}$, Wesley Vieira da Silva ${ }^{[c]}$,

Juliana Cândido Custódio ${ }^{[\mathrm{d}]}$

[a] Doutoranda em Administração pelo Programa de Pós-Graduação em Administração da Pontifícia Universidade Católica do Paraná, Curitiba, PR - Brasil, e-mail: hanna.welgacz@apexbrasil.com.br

[b] Mestre em Administração pelo Programa de Pós-Graduação em Administração da Pontifícia Universidade Católica do Paraná, Curitiba, PR - Brasil, e-mail: isabeladv@bol.com.br

[c] Doutor em Engenharia de Produção pela Universidade Federal de Santa Catarina, professor do Programa de Pós-Graduação em Administração, e-mail: wesley.vieira@gmail.com

[d] Doutoranda em Administração do Programa de Pós-Graduação em Administração da Pontifícia Universidade Católica do Paraná, Curitiba, PR - Brasil, e-mail: juliana.custodio@pucpr.br

\section{Resumo}

Este trabalho tem por objetivo investigar a presença de grupos estratégicos diversificados entre empresas brasileiras. Para evidenciar a existência de diversificação na estratégia das empresas, estudou-se uma amostra de 30 empresas manufatureiras de capital aberto, de 10 setores econômicos, com ações cotadas na Bolsa de Valores de São Paulo. As medidas de diversificação foram os índices de Herfindahl-Hischman, Coeficiente de Entropia e a Relação de Concentração. Para evidenciar a diversificação, os grupos foram representados em relação ao valor da empresa calculado pelo $q$ de Tobin. 0 estudo de natureza quantitativa utilizou dados secundários coletados a partir de bases de dados Economática Softwares para Investimentos LTDA e Relatórios de Informações Anuais (IAN) referentes ao período 2004-2009. Os resultados auferidos demonstram a presença de três grupos de diversificação e evolução dos grupos diversificados rumo a maior índice de diversificação.

Palavras-chave: Estratégia. Diversificação. Concentração.

\section{Abstract}

This study aims to investigate the presence of strategic groups varied between Brazilian companies. To demonstrate the existence of diversification strategies of firms studied a sample of 30 publicly traded manufacturing companies in 10 economic sectors with shares listed on the Bolsa de Valores de Sao Paulo. Diversification measures were the rates of Herfindahl-Hischman, Entropy and Coefficient of Concentration Ratio. To enhance the diversification groups were represented in relation to firm value by Tobin's q calculated. The quantitative study used secondary data collected from databases Economática Software for Investment LTD and Reports Annual 
Information (IAN) for the period 2004-2009. The obtained results demonstrate the presence of three groups of diversification and evolution of diverse groups toward a higher rate of diversification.

Keywords: Strategy. Diversification. Concentration.

\section{Introdução}

O interesse pela estratégia de diversificação não é recente. A princípio, a diversificação em produtos e mercados pode ser vista com naturalidade ao se ver a história do desenvolvimento mercantil da humanidade. Durante milênios o processo de troca comercial entre nações incluía busca de novos produtos e descoberta de novas terras. À primeira vista, pode parecer benéfico introduzir novidades e abrir mercados antes inexplorados para aumentar a receita da empresa.

No entanto, nas últimas décadas pesquisas empíricas em administração conduzidas com rigor científico revelaram peculiaridades em comportamento das empresas diversificadas. A estratégia de diversificação compreende introdução de novos produtos ou entrada em novos mercados, que podem estar ou não relacionados com os negócios vigentes da empresa.

Pesquisas sobre diversificação em economias avançadas (notadamente nos Estados Unidos) demonstraram que empresas diversificadas possuíam valor menor de mercado se forem comparadas com empresas focadas em poucos ou um único negócio. Em função dessa premissa, as empresas deveriam buscar concentrar suas atividades em torno dos principais negócios, sendo que esta atitude aumentaria o valor da empresa. No entanto, em economias em desenvolvimento há evidências de que o comportamento pode ser até inverso.

De acordo com esta linha de argumentação, se torna interessante verificar a presença de grupos estratégicos diversificados no contexto brasileiro. Para isso, coloca-se como objetivo desse trabalho verificar a existência de níveis diferenciados de diversificação entre empresas brasileiras manufatureiras de capital aberto no período de 2004 a 2009.

Como justificativa acadêmica e empírica para o desenvolvimento da presente pesquisa, apresenta-se a razão de que alguns estudos empíricos foram conduzidos para verificar a presença de diversificação em empresas latino-americanas e, em particular, brasileiras. Porém, os resultados não foram conclusivos, e não abrangeram períodos de estudo recentes.
Para Lins e Servaes (2002), custos e benefícios nestes mercados podem não ser os mesmos que os dos países desenvolvidos. A diferença em maturidade dos mercados pode, em parte, explicar o comportamento distinto das empresas norte-americanas, por exemplo, do desempenho das empresas brasileiras.

Nesse sentido, busca-se responder ao seguinte questionamento de pesquisa: será que existem evidências da presença de grupos estratégicos diversificados em empresas de capital aberto brasileiras?

0 presente trabalho está dividido em quatro seções, além desta introdução. Na seção dois se apresenta o referencial teórico-empírico. Em seguida, na terceira seção, é demonstrada a metodologia utilizada. A quarta seção apresenta os resultados obtidos e, por último, na quinta seção, se fazem as considerações finais a respeito da pesquisa empreendida.

\section{Referencial teórico-empírico}

Esta seção inicia-se expondo conceitos e características da estratégia de diversificação, introduz-se a variação de níveis de diversificação de baixos a elevados. A discussão prossegue com a apresentação das razões e motivações para desenvolver diversificação em estratégias das empresas. Em seguida, têm-se a descrição das medidas de diversificação, tecendo ainda considerações sobre as questões de diversificação no contexto das empresas brasileiras e em economias de outros mercados emergentes.

\section{A estratégia de diversificação: conceitos e características}

A estratégia de diversificação é conceituada por Hitt, Ireland e Hoskisson (2007) como opção da empresa em operar negócios em diversas indústrias ou mercados de produtos. Entende-se aqui que a estratégia diversificada envolve tanto termos de oferta de produtos como de localizações geográficas, por exemplo, mercados de distribuição domésticos e internacionais. 
O trabalho de Rumelt (1974 apud CHATTERJEE; WERNERFELT, 1991) foi um dos primeiros a pesquisar a estratégia de diversificação em empresas norte-americanas. Seu trabalho caracterizou o grau de diversificação e introduziu o conceito de diversificação relacionada, quando existem recursos compartilhados entre os negócios. A contribuição desse autor está também na pesquisa do impacto da diversificação na performance das empresas. A taxonomia proposta por Rumelt explica que empresas com diversificação relacionada possuem performance superior às empresas com diversificação não relacionada.

A estratégia de diversificação pode assumir diversos níveis de diversificação, de baixos a elevados níveis. A classificação proposta por Rumelt (1974) define cinco categorias de negócios de acordo com níveis crescentes de diversificação. Uma empresa que possui baixo nível de diversificação concentra seus esforços num único negócio ou em um negócio dominante. Uma empresa é classificada como um único negócio quando mais de $95 \%$ das receitas vêm de um único negócio. Negócios dominantes são empresas que geram entre 70\% e 95\% das receitas de uma única categoria.

Além dos tipos de negócio caracterizados como único e dominante, para empresas mais diversificadas existe diferenciação em relação à conexão entre atuais negócios e novos. Rumelt (1974) define que um novo negócio pode ser relacionado de diversas maneiras com o negócio atual da empresa, e esta relação vai produzir um grau de diversificação relacionada ou não relacionada.

Neste sentido, diversificação relacionada é caracterizada pelo compartilhamento de recursos entre negócios, como mercados comuns, canais de distribuição, tecnologias que são utilizados em proveito comum. Dessa maneira, os negócios criam um para outro um valor adicionado. Já uma diversificação não relacionada pressupõe que os produtos e mercados novos da empresa não possuem ligação com os negócios atuais.

As empresas diversificadas terão níveis de diversificação de moderados a elevados quando existem vínculos entre negócios. Quando não há vínculos comuns entre os negócios, as empresas são tratadas como nível de diversificação elevado. Nestes casos, menos de $70 \%$ das receitas vêm dos negócios dominantes.

Nesse sentido, entende-se que a diversificação relacionada aproveita a sinergia gerada pelo compartilhamento de fontes ou recursos. As habilidades estendidas para novos negócios podem gerar aumento da competência da empresa. A diversificação pode acontecer quando se possui recursos, capacidades e competências essenciais em excesso que tenham múltiplos usos (MONTGOMERY, 1982).

É importante destacar que decisões da estratégia de diversificação são subordinadas à estratégia corporativa que, por sua vez, é diferente e superior à estratégia de negócios. Hitt, Ireland e Hoskisson (2007) distinguem dois níveis de estratégia: uma de nível de negócios (ou competitiva) e outra de nível corporativo, que abrange a companhia inteira. Como esclarecem estes autores, em empresas diversificadas, cada unidade de negócios elege uma estratégia de nível de negócios a ser implementada para atingir competitividade estratégica e ganhar retornos acima da média. Ao mesmo tempo, para empresas diversificadas se torna essencial a elaboração da estratégia que se preocupe com a escolha e administração de seus negócios.

Uma definição formal da estratégia de nível corporativo propõe que "é uma ação posta em prática para ganhar uma vantagem competitiva por meio de escolha e administração de um composto de negócios que competem em diversas indústrias e mercados de produtos" (MONTGOMERY, 1994).

A diversificação pode assumir diversas formas, isto é, a decisão de diversificar pode buscar diversos meios para incorporar novos produtos/serviços e mercados. Grandes empresas diversificadas podem competir entre si em diversos mercados, comportamento que recebe nome de competição multiponto. Integração vertical também é uma forma de diversificação criada para explorar a fatia de mercado e ganhar poder em relação de competidores e Rumelt (1974) classifica empresas com esta característica como categoria dominante vertical (HITT; IRELAND; HOSKISSON, 2007, p. 232). Outra forma de diversificação pode se dar por meio de aquisições (RAMANUJAM; VARADARAJAM, 1989), sendo que aquisição resulta tanto na estratégia de diversificação relacionada como a não relacionada.

Chatterjee e Wernerfelt (1991) exploram a diversificação por meio da temática de entrada em novos mercados. Esses autores classificam os tipos de mercado que a empresa escolhe para entrar e o perfil de recursos que ela possui. Dessa maneira, consideram-se três tipos de recursos: físicos, intangíveis e financeiros. Os dois primeiros são inflexíveis, sendo que poderiam ser utilizados somente em mercados muito relacionados com o negócio atual. Já os recursos financeiros são bastante flexíveis e podem ser utilizados para qualquer tipo de diversificação, tanto relacionada como não relacionada. 0 estudo empírico conduzido por estes 
autores indica que será escolhido aquele mercado que oferecer vantagem competitiva para a firma.

Razões para diversificação: motivação e incentivos

As empresas aumentam a quantidade e tamanho de negócios por diversas razões. A pesquisa pode ser dividida em: i) motivos para melhorar a competitividade estratégica e ii) motivos administrativos. Outra questão são os incentivos para diversificar, pois incentivos são opções para escolha, que pode ser aceita ou não (HITT; IRELAND; HOSKISSON, 2007). A seguir são apresentados estes itens em detalhes explicativos.

Motivos para melhorar a competitividade estratégica são aqueles que permitem criar valor por meio de administração de negócios sob a gestão da estratégia corporativa que se prove como mais vantajosa (retornos mais altos) do que administração de cada unidade de negócios separadamente. Além de melhorar a performance, a estratégia de diversificar pode ser usada para neutralizar o poder de mercado dos concorrentes ou reduzir riscos do negócio.

Nesse sentido, a diversificação com intuito de aumentar a competitividade é implementada visando:

a) economia de escopo: economias de custo atribuídas à transferência das capacidades e competências desenvolvidas em um negócio para novos negócios. Neste momento, acontece compartilhamento de atividades e transferência de competências, onde a diferença entre os dois está em como recursos distintos são (HITT; IRELAND; HOSKISSON, 2007; PORTER, 1986);

b) economia de escala: redução de custos médios e aumento de volume produzido (PORTER, 1986);

c) poder de mercado: imposição de preços, natureza dos produtos no mercado existente por uma empresa (MONTGOMERY, 1985);

d) redução dos riscos: com diversificação de receitas diminui a variabilidade nos lucros (AMIT; LIVNAT, 1988);

e) estrutura de capital e alocação de capital interno: empresas maiores em função de diversificação obtêm acesso ao mercado de capitais e reduzem o risco de falência (BERGER; OFEK, 1995).

Outras razões que dizem respeito a motivações dos administradores que revelam que uma empresa possa implementar a diversificação: a) para reduzir o risco de emprego administrativo, um executivo persegue as próprias metas em vez de optar por melhor opção para o acionista, sendo este raciocínio discutido no trabalho de Jensen e Meckling (1976);

b) com diversificação e crescimento da empresa, aumenta a remuneração dos executivos. Mas, mesmo que os administradores possam ser determinados a aumentar a diversificação, existem estruturas de governança para oprimir esse tipo de comportamento (HITT; IRELAND; HOSKISSON, 2007).

Os incentivos são uma forma de escolher entre seguir a estratégia de diversificação ou optar por permanecer no foco do negócio. Uma legislação governamental antitruste e outras leis fiscais podem contribuir para opção de diversificação. Por outro lado, baixo desempenho pode se configurar como um incentivo para diversificação. Mesmo que existam evidências de que baixos retornos estão relacionados com altos níveis de diversificação, algumas empresas assumem riscos de diversificar (GRANT; JAMMINE, 1988).

A Figura 1 demonstra que pode existir uma relação curvilínea entre a diversificação e o desempenho, mensurado em termos de faturamento, lucro ou mesmo $q$ de Tobin, tal como será usado neste trabalho. Isto é, até determinado nível, as diversificações se revelam compensadores em retornos, posteriormente, começam haver períodos de desinvestimento. Adner e Zemsky (2006) argumentam que as empresas administram a escolha do escopo horizontal ou até quando a diversificação é rentável a partir de trade-off entre a ameaça de perda de foco e a oportunidade de crescimento.

\section{Medidas de diversificação}

Sobre a etimologia do termo "diversificação", Pitts e Hopkins (1982) observam a raiz da palavra que realmente leva a entender que se trata de algo que difere, algo diverso, diferente. Um dos primeiros questionamentos desses autores recai sobre a questão de que aspectos devem ser analisados para que uma empresa seja classificada como diversificada. Na opinião desses autores, qualquer empresa que possui várias áreas funcionais, como marketing, finanças, etc., oferece algum grau de diversificação.

No entanto, como pode ser visto em representativo número de estudos (RUMELT, 1982; MONTGOMERY, 


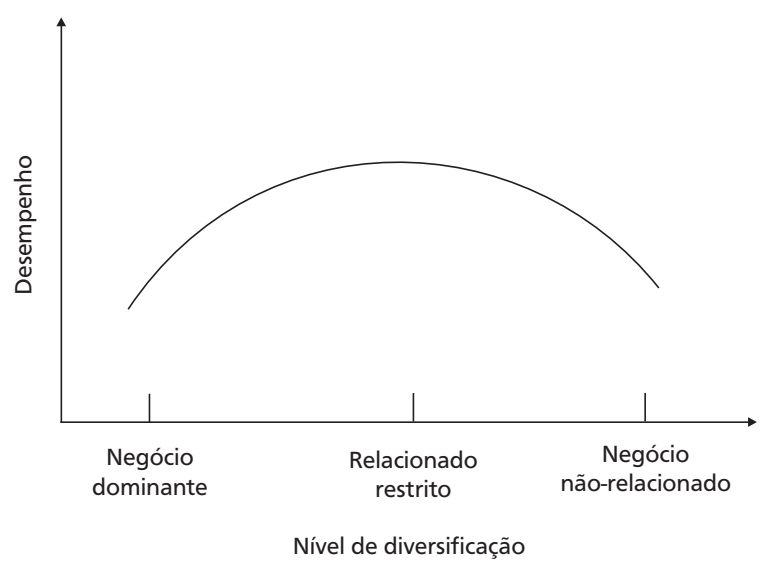

Figura 1 - Relação curvilínea entre diversificação e desempenho.

Fonte: PALICH; CARDINAL; MILLER, 2000.

1982; PITTS; HOPKINS, 1982; PALEPU, 1985), a unidade de medida de diversificação utilizada não leva o termo no seu sentido literal. Diversificação é entendida aqui como um vocábulo do pensamento estratégico. Estratégia de diversificação considera a extensão de negócios que permite aumentar receitas ou diminuir custos visando a ganhos em termos de competitividade.

Como destacam Ramanujam e Varadarajan (1989), as medidas de diversificação de produtos não apresentam consenso entre os estudiosos sobre essa temática. Dois tipos de medidas são mais comumente utilizados com vistas a mensurar o grau de diversificação e o tipo de estratégia de diversificação. Na concepção de Hall Junior e John (1994), as medidas de diversificação podem ser geralmente classificadas dentro de três grupos: medidas contínuas, medidas categóricas e híbridas.

As medidas contínuas auxiliam na quantificação da extensão da diversificação. Montgomery (1982) descreve o sistema de classificação industrial utilizando a Standard Industrial Classification (SIC), nos Estados Unidos. A SIC é um sistema numérico para classificar todos os tipos de atividades econômicas. Cada empresa classifica suas atividades primárias de acordo com essa listagem. No Brasil, Grzebieluckas (2007) descreve o sistema de classificação industrial chamado de Classificação Nacional de Atividade Econômica (CNAE), que, segundo a Resolução IBGE/ CONCLA n. 1, de 25/06/1998, é atualizada mediante a Resolução CONCLA n. 7, de 16/12/2002.

As medidas categóricas são utilizadas com o objetivo de refletir o tipo de diversificação estratégica: relacionada ou não relacionada (RUMELT, 1974; MONTGOMERY, 1982). As medidas híbridas, por sua vez, combinam as medidas contínuas e categóricas (HALL JUNIOR; JOHN, 1994).

É interessante observar as evidências empíricas dos mercados emergentes com a história de diversificação das companhias industriais norte-americanas. A composição empresarial nos países latino-americanos é distinta das formas organizacionais das economias avançadas.

Nas economias em desenvolvimento é frequente o formato de grupos empresariais governados por famílias. A pesquisa de Khanna e Rivkin (2001) demonstra que grupos econômicos são importantes em economias emergentes e moldam padrões de desempenho econômico. Esse fenômeno organizacional que pode ser entendido como sinônimo da diversificação estratégica demonstrou não estar negativamente relacionado com o valor das empresas em 11 dos 14 países emergentes pesquisados por esses autores. Os autores pesquisaram uma amostra de grupos econômicos de 14 países (Brasil, Argentina, Chile, Índia, Indonésia, Israel, México, Peru, Filipinas, África do Sul, Coreia do Sul, Taiwan, Tailândia e Turquia). A premissa estabelecida em função da diversificação ocorrida nas empresas norte-americanas não pôde ser generalizada em 11 dos 14 países. Ou seja, na grande maioria dos países estudados o comportamento da diversificação contrariava a tendência das empresas norte-americanas.

No contexto latino-americano, evidências empíricas para o reflexo da estratégia de diversificação no valor da empresa não possuem resultados uniformes. 
No Brasil, o trabalho de Rogers, Mendes-da-Silva e De Paula (2005), conduzido no período de 1997-2001 com empresas manufatureiras de capital aberto, concluiu que existe associação entre diversificação e o valor de empresas, sendo a configuração dessa relação em forma de "U".

A explicação para a relação não uniforme entre custos e benefícios da diversificação em mercados emergentes pode assumir dimensões diferentes por razões de imperfeição dos mercados serem mais severas (LINS; SERVAES, 2002). Exemplificam os autores que a sobrevivência de firmas mais focadas é mais difícil por conta de sanções contratuais, da relação dos negócios com o governo, produtos e mercados.

\section{Metodologia}

\section{Coleta dos dados}

Foram coletadas empresas listadas na Bolsa de Valores de São Paulo e com sede no Brasil. A população pesquisada refere-se a um total de 438 ações negociadas na Bolsa de Valores de São Paulo, enquanto a amostragem utilizada nesse estudo refere-se a um processo amostral não probabilístico por acessibilidade. No que tange à amostra coletada, inicialmente foram selecionadas um total de 81 ações a partir da disponibilidade dos dados das empresas industriais de capital aberto. Após avaliar a presença daquelas ações que continham todas as informações disponíveis na Base de Dados, chegou-se a uma amostral final de 30 ações.

Dentro da classificação por atividade foram contempladas somente empresas manufatureiras dos setores: têxtil, agro e pesca, alimentos e bebidas, eletroeletrônicos, máquinas industriais, minerais não metálicos, papel e celulose, siderurgia e metalurgia, química e veículos e peças.

A composição das séries temporais compreendeu dados referentes ao período de 2004 a 2009. Ao todo foram incluídas empresas de tamanhos diferenciados, entre médias e grandes. Para amostra final foram consultadas empresas somente com situação ativa na BOVESPA, com sede no Brasil e que emitiram ações tanto ordinárias como preferenciais (não incluindo empresas que possuem ações com desdobramento em classes).

Os dados foram coletados a partir do banco de dados da Economática ${ }^{\circledR}$ Softwares para Investimentos LTDA, tais informações referem-se ao valor das ações das empresas e ao balanço patrimonial destas séries temporais anuais.

Para a estimativa da variável de diversificação, os dados foram obtidos junto a Comissão de Valores Mobiliários (CVM, 2004) por meio de consulta ao banco de dados eletrônico de Relatórios de Informações Anuais (IAN), que as empresas de capital aberto encaminham anualmente a este órgão.

\section{Método de análise dos dados}

A verificação da existência de grupos diversificados foi efetuada por meio do teste de hipóteses não paramétrico de análise de variância de KruskalWallis, com dados referentes ao valor da empresa e categorias de diversificação.

0 propósito do teste de hipóteses de análise de variância não paramétrico de Kruskal-Wallis é verificar se existem diferenças estatisticamente significativas entre os valores médios das categorias analisadas (HAIR, 2005). Vale notar que esta variável foi operacionalizada por meio do índice de $q$ de Tobin, que mensura o valor da empresa e do índice de especialização - aqui denominado de relação de concentração (RC) - em relação aos três principais produtos da empresa.

A análise de variância não paramétrica de KruskalWallis refere-se a um teste de hipóteses por postos que avalia se existem diferenças entre os valores categóricos pesquisados, e a sua escolha se deu em função dos dados coletados não atenderem aos pressupostos de normalidade e homogeneidade de variâncias. Esta técnica já foi utilizada com este propósito nos estudos de Pandya e Rao (1998), Montgomery e Singh (1984) e outros.

Também foram estimados os índices de HerfindahlHisrchman e Coeficiente de Entropia, com o objetivo de mensurar o grau de diversificação dos negócios, ambos em relação aos três principais produtos participantes nos faturamentos das empresas. Na sequência propõe-se uma explanação a respeito das variáveis de valor da empresa e de diversificação.

\section{Variável de medida de valor da empresa - $q$ de Tobin}

$0 q$ de Tobin é definido como o quociente entre o valor de mercado de uma empresa e o valor de reposição de seus ativos físicos, conceito proposto originalmente 
por Tobin e Brainard (1968) e Tobin (1969). Este se consolidou como uma variável com diversas aplicações, notadamente em economia e finanças (FAMA; BARROS, 2001). Nota-se que, em alguns casos na literatura, se pesquisou a influência da estratégia de diversificação dos negócios no valor da empresa, buscando-se utilizar o $q$ de Tobin como uma medida de performance (LANG; STULZ, 1994; BERGER; OFEK, 1995).

Na presente pesquisa optou-se por utilizar a aproximação de cálculo de Chung e Pruitt (1994) devido à disponibilidade de dados para amostra nos balanços financeiros das empresas pesquisadas. Já a medida de aproximação foi utilizada neste trabalho tal como nos trabalhos desenvolvidos por Rogers, Mendes-daSilva e De Paula (2005). Chung e Pruitt (1994) propuseram um cálculo aproximado de $q$ de Tobin, dado por equação (1):

$$
q=\left(\frac{V M O+V M A P+D I V T}{A T}\right)
$$

Onde: VMO refere-se ao valor de mercado das ações ordinárias; VMAP: valor de mercado das ações preferenciais; DIVT: valor contábil das dívidas de curto e longo prazo menos o ativo circulante, após a exclusão do valor dos estoques; e AT: valor contábil do ativo total.

Conforme observado por Fama e Barros (2001), o desafio de se estimar o quociente disposto em (1) consiste na dificuldade em se obter informações a respeito do valor de mercado exato de cada título de dívida. Com isso, a partir do conceito originalmente proposto por Tobin e Brainard (1968) e Tobin (1969), surgiram diversas metodologias do cálculo para esse índice. Dentre os procedimentos que simplificam o cálculo, em parte com propósito de diminuir o esforço computacional, destaca-se o método de Lindenberg e Ross (1981); e o método de Chung e Pruitt (1994), que é uma tentativa de simplificar a aplicação devido a limitações no acesso aos dados.

Variável de medida de diversificação dos negócios

A mensuração da diversificação fornece os elementos para constatar a diversificação de negócios dentro de uma empresa e que venha a permitir verificar a correlação com o valor da empresa. Os indicadores escolhidoslevamem consideraçãoaproduçãoevendas dos produtos comercializados por cada empresa.

Os dados sobre diversificação foram coletados a partir de Relatórios de Informação Anual. Consultou-se a informação referente ao percentual que a receita líquida dos principais produtos representa sobre o total da receita da empresa. 0 período anual consultado está compreendido entre 2004 e 2009.

Os diversos métodos conhecidos para se determinar o grau de concentração revelam características específicas da situação estudada. Nota-se que cada indicador reflete aspectos específicos, o uso complementar de diversas medidas fornece uma análise mais detalhada dos resultados obtidos. As medidas utilizadas nos trabalhos sobre diversificação de negócios são a razão ou relação de concentração (ROGERS; MENDES-DA-SILVA; DE PAULA, 2005; GRZEBIELUCKAS; 2007); coeficiente de entropia de Theil e o índice de Herfindahl-Hirschiman (ROGERS; MENDES-DA-SILVA; DE PAULA, 2005).

\section{Relação de concentração}

Refere-se ao cálculo do índice de especialização de Pandya e Rao (1998), que reflete a razão de concentração das vendas de produtos no total do faturamento da empresa. Mede-se a participação de um, dois ou três produtos principais no faturamento total da empresa.

\section{Índice de Herfindahl-Hirschiman}

O valor de HHI é maximizado quando a participação de um único produto no faturamento da empresa atinge $100 \%$ e, nesses termos, $\mathrm{HHI}=10.000$. Quando os produtos têm participação igualitária, o índice assume valor zero e é mensurado por meio da expressão algébrica (2).

$$
\mathrm{HHI}=\sum_{\mathrm{i}=1}^{3}\left(\frac{\mathrm{p}_{\mathrm{i}}}{\mathrm{p}} \times 100\right)^{2}
$$

\section{Coeficiente de entropia de Theil}

No caso do uso desse indicador, percebe-se que se existir apenas um produto, $\mathrm{CE}=0$; quando todos 
os produtos apresentam igual participação no faturamento da empresa, a entropia é maximizada caso $\mathrm{CE}=\log \mathrm{n}$. Tal coeficiente por ser mensurado por meio da expressão algébrica (3).

$$
C E=\sum_{i=1}^{3} P_{i} \times \log \frac{1}{P_{i}}
$$

Vale salientar que nesta pesquisa o valor de CE é maximizado quando $\mathrm{CE}=\log 3 \sim 0,47712$. A partir da coleta de dados com informações dos principais três produtos, partiu-se para os testes de classificação dos grupos de empresas de estratégias de diversificação por meio de relação de concentração.

\section{Apresentação e análise dos resultados}

O número de empresas por categoria de estratégia de diversificação encontra-se na Tabela 1. Foi utilizada a tipologia de especialização descrita em Pandya e Rao (1998), que considera como estratégia de produto único quando as vendas do primeiro produto representam 95\%; o índice de especialização entre $50-95 \%$, que é caracterizado como uma diversificação moderada; e com índice menor que 50\%, que é visto como uma diversificação alta.

A partir da classificação definida anteriormente, optou-se em estudar o grupo de 3 produtos, que apresentou uma amostra equilibrada em relação aos grupos com 1 e 2 produtos, respectivamente. Tal procedimento foi utilizado nos trabalhos de Grzebieluckas (2007) e Rogers, Mendes-da-Silva e De Paula (2005), nos quais os autores justificam que a quantidade de firmas com estratégia de diversificação moderada e altamente diversificada seria muito reduzida para prosseguir com a pesquisa.

No entanto, o estudo de Grzebieluckas (2007) utilizou um único produto, enquanto os trabalhos desenvolvidos por Rogers, Mendes-da-Silva e De Paula (2005) avaliaram três produtos. A utilização de índices propostos por Pandya e Rao (1998) se justifica pelo fato de obter grupos homogêneos em relação ao número de empresas, pois a contagem leva em consideração os produtos. A diferença do índice do Rumelt consiste em classificação das empresas moderadamente diversificadas - $95 \%$ a $70 \%$ das vendas com o principal negócio, enquanto na classificação de Pandya e Rao (1998) este índice foi de $95 \%$ a $50 \%$.

Cabe destacar que o uso de categorias é mais comum em pesquisas científicas do que a contagem de dígitos para setores econômicos ou sistema SIC norte-americano, (PANDYA; RAO, 1998). Montgomery (1982) obteve resultados similares com ambos os métodos e ainda considerou que as medidas categóricas de Rumelt podem ser utilizadas para mostrar a ligação entre diversificação e performance.

Posteriormente, realizou-se um teste visando identificar e tratar os potenciais outliers. Hair (2005) sugerem levar em conta o intervalo de confiança de 2,5 desvios padrão. A amostra coletada constitui-se conforme evidencia a Tabela 2.

Vale salientar que o critério estabelecido para a composição da amostra coletada foi considerar apenas as empresas que apresentassem seis períodos consecutivos no período compreendido entre 2004 e 2009.

Tabela 1 - Classificação dos grupos de empresas de acordo com o percentual de receita líquida

\begin{tabular}{lccc}
\hline Categoria & $\begin{array}{c}\text { 1 produto, quantidade de } \\
\text { empresas }\end{array}$ & $\begin{array}{c}\text { 2 produtos, quantidade de } \\
\text { empresas }\end{array}$ & $\begin{array}{c}\text { 3 produtos, quantidade de } \\
\text { empresas }\end{array}$ \\
\hline Negócio único: IE > 95\% & 11 & 25 & 36 \\
$\begin{array}{l}\text { Diversificação moderada: IE } \\
\text { entre } 50 \text { e } 95 \%\end{array}$ & 23 & 34 & 25 \\
$\begin{array}{l}\text { Diversificação elevada: IE me- } \\
\text { nos de 50\% }\end{array}$ & 36 & 15 & 12 \\
Total de observações & 70 & 74 & 73 \\
\hline
\end{tabular}

Nota: Levou-se em conta os três principais produtos das empresas pesquisadas. 
Análise descritiva dos dados coletados

Foram identificados três grupos de empresas divididas de acordo com seu grau de diversificação em função da Relação de Concentração (RC), conforme pode ser visto na Tabela 2. Por outro lado, os resultados dispostos na Tabela 3 evidenciam a média de $q$ de Tobin por categoria.

A estratificação das empresas classificadas em função ao índice de relação de concentração (RC) demonstra que a quantidade de observações das 30 empresas por grupo estratégico apresenta $50 \%$ das observações na categoria do negócio único. A média geral de valor do $q$ de Tobin para todas as empresas ficou em 1,71 .

A Figura 2 visualiza as medidas de $q$ de Tobin para cada classificação de diversificação identificada na Tabela 3. As empresas que foram classificadas como negócio único apresentaram média do valor $q$ de 1,46; as empresas classificadas com diversificação moderada, 1,81; e empresas com diversificação alta apresentaram média do valor $q$ de Tobin de 2,35.

Observa-se que a média geral para todas as empresas ficou em 71\%. 0 trabalho de Rogers, Mendesda-Silva e De Paula (2005) constatou para a amostra de 1997 a 2001 a média de $91 \%$ de faturamento com três principais produtos. Os dados permitem a observação de que a diversificação está mais presente na estratégia das empresas, pois no período estudado de 2002 a 2007 as empresas obtiveram 71\% do faturamento com três principais produtos, enquanto no passado este índice era de $91 \%$.

Observação do valor do $q$ de Tobin na Tabela 3 mostra a tendência de aumentar acompanhando o aumento da diversificação da empresa. 0 grupo de empresas altamente diversificadas (15\% da amostra) possui valor de $q$ mais alto do que o grupo de observações com negócio único - valor de $q$ de 1,46.

\section{Resultado da análise de variância não paramétrica}

Visando verificar se existem diferenças estatisticamente significativas entre os grupos estratégicos caracterizados como diversificados, realizou-se o teste de hipóteses de análise de variância não paramétrico de Kruskal-Wallis. A Figura 2 mostra o comportamento descritivo dos grupos de diversificação em relação ao $q$ de Tobin.

Foram efetuados testes de hipóteses de análise de variância de Kruskal-Wallis visando ratificar a hipótese de que existem diferenças estatisticamente significativas entre os grupos diversificados. Considerando um nível de significância de 1\%, deve-se rejeitar a hipótese de que as médias de $q$ são iguais entre os grupos de diversificação, tal como pode ser observado na Tabela 4.

Tabela 2 - Amostra constituída de 30 empresas nos seis anos consultados

\begin{tabular}{lr}
\hline Categoria & Amost \\
\hline Negócio único & 90 \\
Diversificação moderada & 63 \\
Diversificação elevada & 27 \\
Total & 180
\end{tabular}

Tabela 3 - Médias de q de Tobin dos grupos estratégicos em relação ao faturamento com três principais produtos

\begin{tabular}{lcccc}
\hline Grupo (RC) & Amostras & \% da amostra & \% diversificação & $q$ Tobin \\
\hline Negócio único (>95\%) & 90 & 50 & 0,99 & 1,46 \\
Diversificação moderada (50\%-95\%) & 63 & 35 & 0,83 & 1,81 \\
Diversificação elevada (<50\%) & 27 & 15 & $\mathbf{0 , 3 0}$ & $\mathbf{2 , 3 5}$ \\
Total & $\mathbf{1 8 0}$ & $\mathbf{1 0 0}$ & $\mathbf{0 , 7 1}$ & $\mathbf{1 , 7 1}$ \\
\hline
\end{tabular}


Pautando-se na tabela anterior, é possível afirmar que as empresas mais diversificadas possuem um maior valor de $q$ de Tobin. 0 aspecto evidenciado na Figura 3 é distinto do comportamento das empresas norte-americanas observado por Palich, Cardinal e Miller (2000), no qual foi sugerido o modelo em formato de "U" invertido.

\section{Evolução das categorias de diversificação} no período de 2004- 2009

Com base nos resultados auferidos da Figura 3, tem-se a evolução da diversificação dos grupos estratégicos a partir do índice de relação de concentração estimado para os três principais produtos.

Observa-se com base na Figura 3 que, no ano de 2004, a média de diversificação foi de aproximadamente 0,87 , o que pode ser interpretado como o

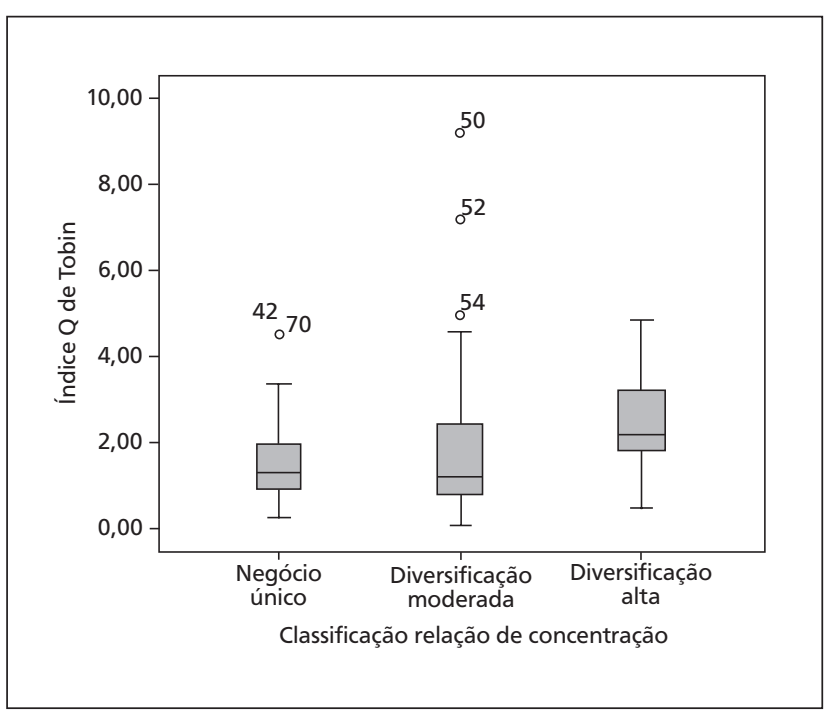

Figura 2 - Boxplot dos grupos de diversificação em relação ao $q$ de Tobin

Fonte: Resultados extraídos do SPSS.

Tabela 4 - Estatística Kruskal-Wallis para q de Tobin e relação de concentração

\begin{tabular}{lc}
\hline & Índice $q$ de Tobin \\
\hline Chi-Square & 12,592 \\
DF & 2 \\
P-value & 0,001 \\
\hline
\end{tabular}

Fonte: Resultados Extraídos do SPSS. fato das empresas, em média, obterem com seus três principais produtos cerca de $87 \%$ do faturamento.

Percebe-se que no decorrer do período pesquisado este indicador se reduz, porém, no ano de 2009, as empresas obtiveram com os seus três principais produtos um valor menor, ou seja, em torno de $82 \%$ do faturamento. Este fato evidencia que as empresas pesquisadas estão se tornando mais diversificadas, já que engajam uma maior quantidade de produtos no mercado procurando obter seu faturamento total.

\section{Considerações finais}

Estratégia de diversificação é uma temática muito pesquisada e com um vasto referencial teórico-empírico. Em economias desenvolvidas foram empreendidas diversas pesquisas empíricas com o intuito de verificar a presença de grupos estratégicos nas atividades das empresas e sua relação com o desempenho, valor da empresa, entre outros indicadores.

No entanto, é fácil perceber a partir da convergência de opiniões na literatura pesquisada da área de estratégia que, dependendo do grau de maturidade da economia estudada, a diversificação dentro das empresas encontra-se presente em maior ou menor grau.

0 presente trabalho teve como objetivo avaliar a presença de grupos estratégicos diversificados na economia brasileira a partir de amostra de empresas manufatureiras de capital aberto. Verificou-se, com base nos resultados auferidos da pesquisa, que no período estudado de 2004 a 2009 existem evidências de níveis diferenciados de diversificação entre as empresas brasileiras de capital aberto.

Nesse sentido, pode-se afirmar que a questão de pesquisa foi integralmente respondida. Tal assertiva pôde ser corroborada em função do faturamento com os três principais produtos no ano de 2004, que contribuiu com 87\%, enquanto em 2009 esse índice diminui para $82 \%$. Isto demonstra que mais produtos contribuíram com o faturamento total das empresas pesquisadas.

Os testes estatísticos também evidenciaram confiabilidade nos resultados de identificação dos grupos estratégicos de diversificação: de negócio único $50 \%$ da amostra, de diversificação moderada 35\%, e de diversificação elevada $15 \%$ da amostra. Ainda em relação ao valor da empresa, foi revelada a tendência de as empresas com maior diversificação possuírem maior valor de $q$ de Tobin. 


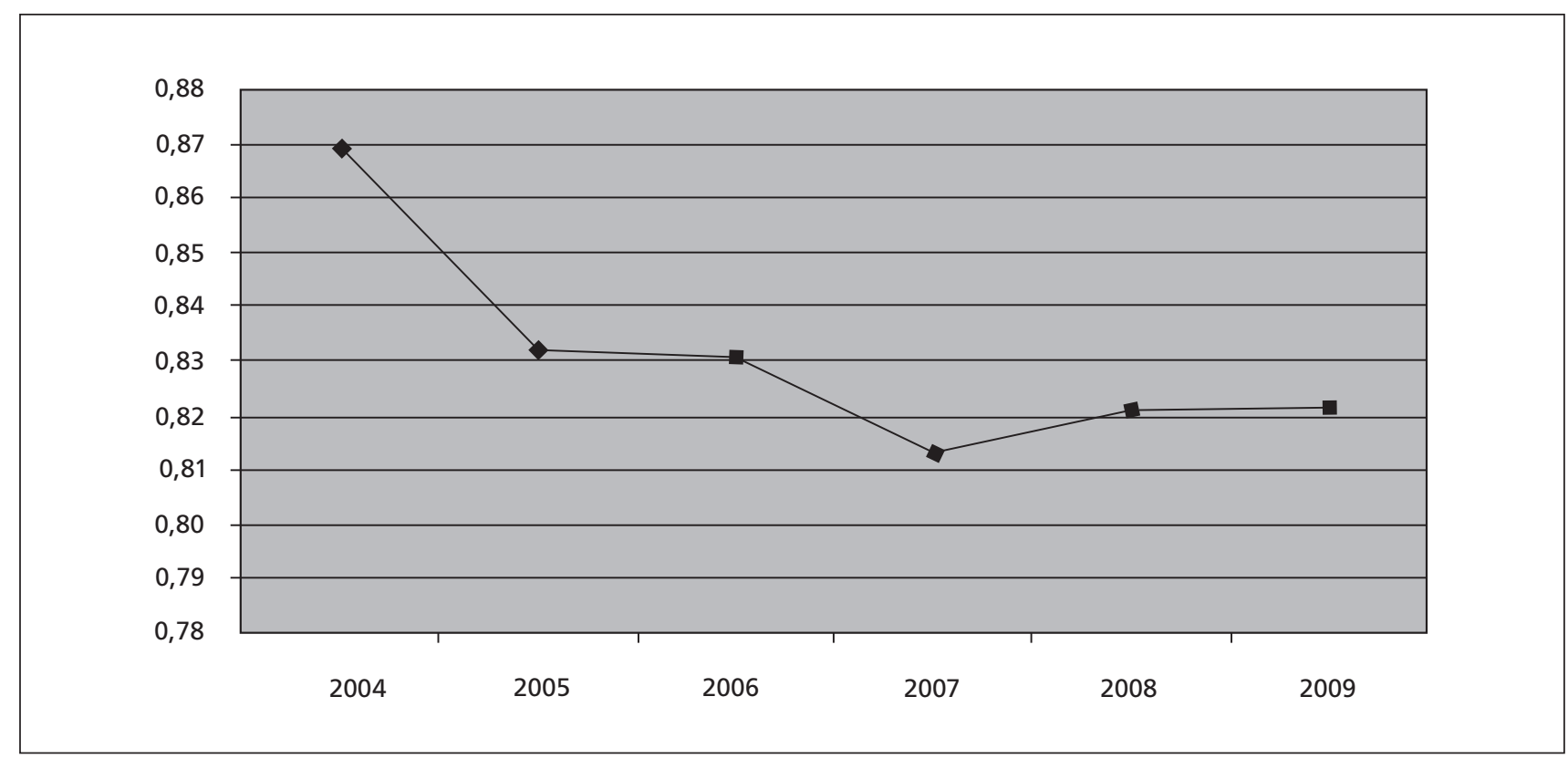

Figura 3 - Evolução das categorias de diversificação no período de 2004-2009

Fonte: Resultados da pesquisa.

Ademais, recomenda-se a realização de outras pesquisas visando verificar a diversificação nas empresas brasileiras por meio de outras medidas como classificação das atividades, de maneira similar como é efetuado nos Estados Unidos, por meio de sistema SIC. Pressupõe-se que proceder com esta metodologia possa levar aos resultados que permitam maior exposição do assunto estudado.

\section{Referências}

ADNER, R.; ZEMSKY, P. A demand-based perspective on sustainable competitive advantage. Strategic Management Journal, v. 27, n. 3, p. 215-239, 2006.

AMIT, R.; LIVNAT, J. Diversification and the risk-return trade-off. Academy of Management Journal, v. 31, n. 1, p. 154-166, 1988.

ANDERSON, R. C. et al. Corporate governance and firm diversification. Financial Management, v. 29, n. 1, p. 5-22, 2000.

BERGER, P. G.; OFEK, E. Diversification's effect on firm value. Journal of Financial Economics, v. 37, n. 1, p. 39-65, 1995.

CHATTERJEE, S.; WERNERFELT, B. The link between resources and type of diversification: theory and evidence. Strategic Management Journal, v. 12, n. 1, p. 33-48, 1991.
CHUNG, K. H.; PRUITT, S. W. A simple approximation of Tobin's q. Financial Management, v. 23, n. 3, p. 70-74, 1994. COMISSÃO DE VALORES MOBÍLIÁRIOS - CVM. Relatórios de Informações Anuais. Rio de Janeiro: Ministério da Fazenda, 2004. Disponível em: <www.cvm.gov.br>. Acesso em: 1ํㅡ. nov. 2009.

FAMA, R.; BARROS, L. A. B. de C. Q de Tobin e seu uso em finanças: aspectos metodológicos e conceituais. In: SEMINÁRIOS EM ADMINISTRAÇÃO DA FEA/USP, 5., 2001, São Paulo. Anais... São Paulo: SEMEAD FEA/USP, 2001.

GRANT, R. M.; JAMMINE, A. P. Performance differences between the Wrigley/Rumelt strategic categories. Strategic Management Journal, v. 9, n. 4, p. 333-346, 1988.

GRZEBIELUCKAS, C. A estratégia de diversificação e sua influência na performance: uma análise empírica em companhias abertas no Brasil. 2007. 144 f. Dissertação (Mestrado em Administração) - Universidade do Vale do Itajaí, Biguaçu, SC, 2007.

HAIR, J. F. Fundamentos de métodos de pesquisa em administração. Porto Alegre: Bookman, 2005.

HALL JUNIOR, E. H.; JOHN, C. H. St. A methodological note on diversity measurement. Strategic Management Journal, v. 15, n. 2, p. 153-168, 1994. 
HITT, M. A.; IRELAND, R. D.; HOSKISSON, R. E. Administração estratégica: competitividade e globalização. São Paulo: Pioneira Thomson Learning, 2007.

JENSEN, M. C.; MECKLING, W. Theory of firm: managerial behavior, agency costs and ownership structure. Journal of Financial Economics, v. 3, n. 4, p. 305-360, 1976.

KHANNA, T.; RIVKIN, J. W. Estimating the performance effects of business group in emerging markets. Strategic Management Journal, v. 22, n. 1, p. 45-74, 2001.

LANG, L.; STULZ, R. Tobin's Q. corporate diversification and firm performance. Journal of Political Economy, v. 102, n. 6, p. 1248-1280, 1994.

LINDENBERG, E.; ROSS, S. Tobin's Q ratio and industrial organization. Journal of Business, v. 54, n. 1, p. 1-32, 1981.

LINS, K.; SERVAES, H. Is corporate diversification beneficial in emerging markets? Financial Management, n. 31, p. 5-31, 2002.

MONTGOMERY, C. A. The measurement of firm diversification: some new empirical evidence. The Academy of Management Journal, v. 25, n. 2, p. 299-307, 1982.

MONTGOMERY, C. A. Product-market diversification and market power. Academy of Management Journal, v. 28, n. 4, p. 789-798, 1985.

MONTGOMERY, C. A. Corporate diversification. Journal of Economic Perspectives, n. 8, p. 163-178, 1994.

MONTGOMERY, C.; SINGH, H. Diversification strategy and systematic risk. Strategic Management Journal, v. 5, n. 2, p. 181-191, 1984.

PALEPU, K. Diversification, strategy, profit performance and the entropy measure. Strategic Management Journal, v. 6, n. 3, p. 239-255, 1985.

PALICH, L. E.; CARDINAL, L. B.; MILLER, C. C. Curvilinearity in the diversification-performance linkage: an examination of over three decades of research. Strategic Management Journal, v. 21, n. 2, p. 155-174, 2000.
PANDYA, A. M.; RAO, N. V. Diversification and firm performance an empirical evaluation. Journal of Financial and Strategic Decisions, v. 11, n. 2, p. 67-81, 1998.

PITTS, R. A.; HOPKINS, H. D. Firm diversity: conceptualization and measurement. The Academy of Management Review, v. 7, n. 4, p. 620-629, 1982.

PORTER, M. Estratégia competitiva: técnicas para análise de indústrias e da concorrência. Rio de Janeiro: Campus, 1986.

RAMANUJAM, V.; VARADARAJAN P. Research on corporate diversification: a synthesis. Strategic Management Journal, v. 10, n. 6, p. 523-551, 1989.

ROGERS, P.; MENDES-DA-SILVA, W.; DE PAULA, G. M. Estratégias corporativas de diversificação e valor das empresas na América Latina: estudo de caso do Brasil. In: ASAMBLEA CONSEJO LATINOAMERICANO DE ESCUELAS DE ADMINISTRACION, 40., 2005. Anais... Santiago do Chile: CLADEA, 2005.

RUMELT, R. Strategy, structure and economic performance. Boston: Harvard, 1974.

RUMELT, R. Diversification strategy and profitability. Strategic Management Journal, v. 3, n. 4, p. 359-369, 1982.

TOBIN, J. A general equilibrium approach to monetary theory. Journal of Money, Credit and Banking, v. 1, n. 1, p. 15-29, 1969.

TOBIN, J.; BRAINARD, W. Pitfalls in financial model building. American Economic Review, v. 58, n. 2, p. 99-122, 1968.

Recebido: 07/02/2010

Received: 02/07/2010

Aprovado: 26/09/2010

Approved: 09/26/2010 